NOTICE: This is the author's version of a work that was accepted for publication in Learning and Instruction. Changes resulting from the publishing process, such as peer review, editing, corrections, structural formatting, and other quality control mechanisms may not be reflected in this document. Changes may have been made to this work since it was submitted for publication. A definitive version was subsequently published in Learning and Instruction, Volume 25, June 2013, Pages 71-84. http://dx.doi.org/10.1016/j.learninstruc.2012.11.005 
Elsevier Editorial System(tm) for Learning and Instruction

Manuscript Draft

Manuscript Number: JLI-D-11-00172R3

Title: Cueing animations: Dynamic signaling aids information extraction and comprehension

Article Type: Research Paper

Keywords: Animation, Animation Processing Model, Event cueing, Cue obedience, Eye tracking, Timelocked data.

Corresponding Author: Dr. Jean-Michel Boucheix, Ph.D

Corresponding Author's Institution: LEAD-CNRS UMR 5022

First Author: Jean-Michel Boucheix, Ph.D

Order of Authors: Jean-Michel Boucheix, Ph.D; Richard K Lowe, Ph.D; Dian K Putri, PhD Student; Jonathan Groff, PhD Student

Abstract: The effectiveness of animations containing two novel forms of animation cueing that target relations between event units rather than individual entities was compared with that of animations containing conventional entity-based cueing or no cues. These relational event unit cues (progressive path and local coordinated cues) were specifically designed to support key learning processes posited by the Animation Processing Model (Authors, 2008). Four groups of undergraduates $(\mathrm{N}=84)$ studied a user-controllable animation of a piano mechanism and then were assessed for mental model quality (via a written comprehension test) and knowledge of the mechanism's dynamics (via a novel nonverbal manipulation test). Time-locked eye tracking was used to characterize participants' obedience to cues (initial engagement versus ongoing loyalty) across the learning period. For both output measures, participants in the two relational event unit cueing conditions were superior to those in the entity-based and uncued conditions. Time-locked eye tracking analysis of cue obedience revealed that initial cue engagement did not guarantee ongoing cue loyalty. The findings suggest that the Animation Processing Model provides a principled basis for designing more effective animation support. 


\title{
Cueing animations: Dynamic signaling aids information extraction and comprehension
}

\author{
Jean-Michel Boucheix $^{\mathrm{a}, *}$, Richard. K. Lowe ${ }^{\mathrm{b}}$, Dian. K. Putri ${ }^{\mathrm{a}, \mathrm{c}}$, and Jonathan Groff ${ }^{\mathrm{a}}$ \\ ${ }^{a}$ Lead-CNRS, University of Burgundy, Dijon, France, Jean-Michel.Boucheix@u- \\ bourgogne.fr \\ ${ }^{\mathrm{b}}$ Curtin University, Perth, Australia, r.k.lowe@ curtin.edu.au \\ ${ }^{\mathrm{c}}$ Gunadarma University, Jakarta, Indonesia, dian@ @ staff.gunadarma.ac.id
}

\begin{abstract}
The effectiveness of animations containing two novel forms of animation cueing that target relations between event units rather than individual entities was compared with that of animations containing conventional entity-based cueing or no cues. These relational event unit cues (progressive path and local coordinated cues) were specifically designed to support key learning processes posited by the Animation Processing Model (Authors, 2008). Four groups of undergraduates $(\mathrm{N}=84)$ studied a user-controllable animation of a piano mechanism and then were assessed for mental model quality (via a written comprehension test) and knowledge of the mechanism's dynamics (via a novel non-verbal manipulation test). Time-locked eye tracking was used to characterize participants' obedience to cues (initial engagement versus ongoing loyalty) across the learning period. For both output measures, participants in the two relational event unit cueing conditions were superior to those in the entity-based and uncued conditions. Time-locked eye tracking analysis of cue obedience revealed that initial cue engagement did not guarantee ongoing cue loyalty. The findings suggest that the Animation Processing Model provides a principled basis for designing more effective animation support.
\end{abstract}

Key Words: Animation, Animation Processing Model, Event cueing, Cue obedience, Eye tracking, Time-locked data.

*Corresponding author: Tel.: +33 3803957 65; fax: +33 380395767 .

Postal address: LEAD-CNRS, UMR5022, Université de Bourgogne, Pôle AAFE, Esplanade Erasme, BP 26513, 21065, Dijon-cedex.

E-mail addresses: Jean-Michel.Boucheix@u-bourgogne.fr 


\section{Introduction}

This paper reports a study of learning from an animated depiction of a complex physical system that is new to most people (an upright piano mechanism). Animations of complex content can pose considerable challenges for learners (Bétrancourt, 2005; Fischer, Lowe \& Schwan, 2008; Höffler \& Leutner, 2007) due to attributes such as the amount of information presented and its transience for working memory (Authors, 1999; Ayres and Paas, 2007 a, b; Moreno, 2007; Spanjers, Van Gog, and Van Merrienboer, 2010; Wouters, Paas, and Van Merrienboer, 2008). Compared with static graphics, the distinctive continuous temporal changes that characterize animated displays introduce “... additional and qualitatively different information processing demands" (Authors, 2003, p.157). Learners typically respond to these demands by exercising their visual attention very selectively (Lowe \& Schnotz, 2008).

A fundamental problem facing learners is to extract information from the animation that is of most relevance to their construction of a high quality mental model of the referent subject matter (Authors, 2008). For a mental model representing dynamic content to be of high quality, its construction needs to include not only all key entities, but also how those entities behave (i.e., events) and their interrelations across space and time. Without an effective internal representation of these dynamics and relationships, the result will not be the type of coherent and runnable mental model a learner needs for success in tasks such as answering questions, making inferences or predictions, and solving problems.

With animations about new subject matter, learners are typically heavily reliant on bottom-up processing that is based on the perceptual characteristics of the display because they lack the background knowledge required for more top-down approaches (cf. Kriz \& Hegarty, 2007). Ideally, the visual salience of information displayed should therefore be well aligned with its 
relevance. Unfortunately, as with the piano mechanism example used in the present study, this is not always the case. Although salience-relevance misalignment is not a universal characteristic of animations, it tends to be a feature of technical animations that depict their subject matter in a behaviourally realistic manner (cf. Ploetzner \& Lowe, 2012). In video clips, which are widely used, such misalignments very often occur; and this aspect of visualization was investigated here in the example of animation. When this occurs, learners exercising their selective attention in order to cope with the taxing processing demands can neglect high relevance low salience information in favour of information that is more conspicuous (Schnotz \& Lowe, 2008). The net result of failing to extract crucial high relevance information from an animation is that the quality of the mental model constructed can be compromised.

\subsection{Cueing static and animated graphics}

Cueing is an approach intended to address the problem of information extraction failures by guiding learners to high relevance information. In conventional cueing (as long applied to static graphics), visuospatial contrast is used to alter the perceptibility profile (Authors, 2010) of the entities comprising a display in order to improve the salience-relevance alignment. For example, high relevance aspects might be signaled by introducing a colour contrast that makes them stand out from the rest of the display. The potential of visual cueing to support learner processing of animations has received considerable attention in recent years (Amadieu, Mariné \& Lemay, 2011; Authors, 2005; Authors, 2010a; De Koning, Tabbers, Rikers \& Paas, 2007, 2009, 2010; Jamet, Gavota \& Quaireau, 2008; Kriz, \& Hegarty, 2007; Lin \& Atkinson, 2011; Mautone \& Mayer, 2001 and for a comprehensive review, see De Koning, Tabbers, Rikers \& Paas, 2009). However, research indicates that when conventional visuospatial cueing is applied to animations (rather than static graphics), it too often lacks educational effectiveness (e.g., Authors, 2011). Interestingly, in 6 of the 13 studies examined 
by de Koning et al., 2009 (see De Koning, 2009, p. 126-127) for which there was a positive effect of cueing on learning outcomes (and not merely on attention direction), 5 out the 6 were either visual cues that involved dynamics (Authors, 2010a; Fisher, Lowe \& Schwan, 2008; Fisher \& Schwan, 2008) or verbal cues that highlighted movements and relations within dynamic representations (Authors, 2005; Huk, Steinke \& Floto, 2003). Failures of visuospatial cueing in animated contexts have been attributed to the inability of visuospatial contrast to compete strongly enough for learner attention with the forceful attention directing effect of the dynamic contrast which is present in animations but not in their static counterparts (Authors, 2011).

In order for cueing to compete successfully for attention within an animation, its signaling needs to be strong enough to counter the powerful direction of attention from the animation's own dynamics. Enhancing conventional cueing by supplementing its visuospatial contrast with dynamic contrast (Author, 2003) is one possibility for increasing cue effectiveness within animated contexts. The present research used two forms of cueing that not only recruit dynamic contrast for the purposes of strengthening cueing of entities, but also expand the role of cueing to include explicit signaling of events and relationships. They were designed to facilitate a number of psychological processes posited by the Animation Processing Model (APM) to be required of learners faced with a complex animation of new content (Authors, 2008, 2011). Before detailing the specific processing activities targeted by these two new forms of cueing, we present a summary of the Animation Processing Model.

\subsection{Animation Processing Model and Cueing}

According to the five-phase hierarchical Animation Processing Model, learning from animation is a cumulative process for building dynamic mental models in which events play a 
crucial role. The present study focuses on cues specifically designed to support the earlier activities of animation processing (APM phases 1,2 and 3) which concern the extraction of fundamental information not only about an animation's constituent graphic entities but also the spatio-temporal relationships between them. The APM characterizes phase 1 processing as learner parsing of the animation's continuous flux of dynamic information into individual event units - that is to say, entities plus their associated behaviours. This initial parsing is undertaken during perceptual exploration of the animated display at the local level. The competition for attention associated with this parsing activity can have detrimental effects due to the limited time available for processing (cf. Barrouillet, Bernardin, Portrat, Vergauwe, \& Camos, 2007; Spanjers et al., 2010). The event unit concept that is central to the APM has its origins in the work on event cognition by Zacks and colleagues (e.g., Kurby \& Zacks, 2007). The event units identified during phase 1 processing are highly localized in specific spatial and temporal regions of the animation. In phase 2 , the learner links these local segments into somewhat broader event structures which are termed dynamic micro-chunks. During phase 3, various sets of dynamic micro-chunks are interconnected to form super ordinate spatiotemporal structures. These structures embody the depicted system's causality in the form of causal chains that are responsible for the system's operation (Authors, 2008; Kriz \& Hegarty, 2007).

With conventional visuospatial cues, only the entities that comprise the display are directly targeted. Such cues typically provide no specific guidance about (i) the events those entities engage in, or (ii) the ways entities and events in one part of the display are related to those in other regions. Our approach to cueing does not treat entities in isolation from their behavior, as it is the case with conventional visuospatial cueing. Instead, it targets the event units posited by the APM as being fundamental to animation processing and the relations between 
them that are the basis for hierarchical linking of event units into a mental model. For this reason, we refer to this approach as Relational Event Unit cueing. It is important to emphasize that relational event cueing does not exclude the entities themselves from the signaling - it is clearly important for learners to know which items in the display deserve their special attention (cf. Narayanan \& Hegarty, 1998). Rather, the APM indicates that although the cueing of entities in an animation is necessary, it is not sufficient.

We have devised two forms of relational event unit cueing: Progressive Path cues and Local Coordinated cues. Both of these are dynamic forms of signaling that use directional moving colour to provide stronger direction of attention than would be possible with conventional visuospatial cues. During APM Phase 1 (parsing of the animation to produce event units), aspects of the animation that make them stand out from the rest of the display are preferentially processed. Because the human visual system privileges dynamic information (Wolfe \& Howowitz, 2004) aspects whose behavior contrasts markedly with that of their surroundings receive preferential attention. When a visuospatial cue is used in an animation, the only movement it ever exhibits is that inherited from the entity to which it is applied. Under these circumstances, the cue does not compete with the entity for learner attention but rather is subservient to it. However, the situation with relational event unit cueing is very different. These cues move within and between the entities that are located along the cueing path and this moving colour provides ongoing attention-capturing dynamic contrast that is not present with visuospatial cueing.

The moving colour change that is a feature of relational event unit cueing is also designed to support APM phase 2 and 3 processing. These two phases are concerned with the progressive interconnection of event units identified in APM phase 1 processing, at both a regional level (phase 2, formation of dynamic micro chunks) and more broadly (phase 3, establishing causal 
chains). The intention with relational event unit cueing is that as the moving colour change travels through adjacent event units, it draws attention to the relationships between them. Once noticed, the learner can then cluster these related event units into local chunks, which in turn may be linked into causal chains encompassing the animation as a whole. In this way, relational event unit cues should not only help learners to detect high relevance event units, they should also help in building them into a coherent, hierarchically organized, high quality mental model.

According to the APM, once key event units in the animation have been identified during phase 1 processing, learner activity needs to progress to establishing relations between those event units (phase 2 for regional relations and phase 3 for broader connections). This means that after initial engagement with the cues in order to detect the key event units, learner loyalty to cueing should diminish so that attention can be directed to non-cued materials to which those event units need to be connected.

On one hand, with visuo-spatial cueing, the diminishing of cue loyalty after initial engagement could be beneficial (according to the APM) if learners then shifted their attention to one or more entities to which it was strongly related. However, this depends on learners being able to single out such related entities for themselves. Without further assistance it is unlikely that novices in the depicted domain would be able to identify these correctly.

On the other hand, relational cueing actually indicates to learners which entities are related to which other entities because of the way it explicitly connects them across space and time. And it may be that, for learners, a minimal amount of time is required for an efficient processing of the connection between two or more entities via relational cueing (and its consequences) before moving to other related entities. 


\subsection{Relational event unit cueing}

The main difference between two forms of dynamic relational event unit cueing variants, respectively, Progressive Path cues and Local Coordinated cues is how tightly targeted they are. With progressive path cues, moving colour is applied along the entire length of causal chain paths, whereas with local coordinated cues it is applied to only specific localities where operationally important interactions take place. Progressive path cueing has already been investigated (Authors, 2010a). In this type of cueing, spreading of a causal chain through the components of the mechanism is progressively cued through its time course via high perceptibility directional ribbons of colour overlaid on the depicted material (Figure 2b and Appendix C). The progress of this cueing through the animation's most thematically relevant graphic entities is synchronized with the propagation of the main causal chains via those entities. Different cue colors (red and blue) signal events occurring along the different constituent causal chains of the piano mechanism to indicate operations taking place in parallel. With this technique, there is not only signaling of successive entities along the causal chains, but also dynamic cueing of pathways connecting them.

The prior study found that progressive path cues were superior to no cues and suggested that event cueing was particularly supportive of APM phase 2 processing (i.e., initial linking of entities). However, that study also indicated there could be downsides for progressive path cueing. One potential problem is that all information (entities and events) along the causal chains is equally cued, irrespectively of its functional importance and degree of saliencerelevance alignment. For example, the large and salient hammer and damper behaviors are cued to the same extent as the far less conspicuous events involving the key tail, whippen and spoon. Drawing even more attention to aspects that are already conspicuous could defeat the purpose of cueing. Another potential problem with progressive path cueing is that it could induce indiscriminate cue-following behavior that severely circumscribes learner exploration 
of the display. Such slavish following of a cue through the piano mechanism may therefore impede relation formation and active inferences of the type needed for interconnecting offpath dynamic micro-chunks in APM Phase 3 processing.

To address these potential negative side effects and support more efficient APM phase 3 processing, another more tightly targeted form of event cueing was developed. Local coordinated cues differ from progressive path cues because, rather than being applied to entire causal chains, they are confined to only limited isolated regions that have particular functional significance. Local coordinated cues are applied to only specific localities where operationally important interactions take place (Figure $2 \mathrm{~b}$ and Appendix C). In essence, these two forms of event cueing differ with respect to their scope and targeting. The present study compares the effectiveness of local coordinated and progressive path cueing. It also addresses a possible limitation of the prior study in which progressive path cueing was compared with a no-cue condition but not with entity cueing. The effect of the following four cueing conditions on comprehension of the piano animation were therefore compared: progressive path cues, local coordinated cues, conventional entity-based cues and uncued. This is the first time that relational and dynamic cueing forms are systematically compared to conventional cueing form.

\subsection{Complex animation and cueing}

Comprehensive cueing should be particularly important with animations showing complex dynamic processes such as an upright piano's hidden mechanism, the topic of the animation used in the present study, (Figure 1). This content was chosen because it exemplifies a broad class of very general processing challenges that learners could meet with complex animations having a high degree of behavioral realism. The dynamic mechanism by which a musical note is produced when a pianist presses a key on a piano keyboard essentially consists of a 
sophisticated system of levers and pivots. This mechanical sophistication results in a high degree of operational complexity during which the various components interact in a myriad of subtle and closely interrelated ways. A detailed account of the piano mechanism's operation, a comprehensive explanation of its complexities, and the likely processing challenges that its comprehension poses for learners are given elsewhere (Authors, 2010a).

Insert Figure 1 about here

The components depicted in the piano animation used for the present study compete unequally for the viewer's attention. Both the basic visual properties of these components (an array of varied graphic entities) and their behavior contribute to potentially problematic misalignments of perceptual salience and thematic relevance. For example, the hammer has a high level of perceptibility because it is a large, distinctively-shaped item whose movement is rapid and covers much of the display area. Although the hammer plays a central function in making the string sound, its successful operation depends on parts of the mechanism with far lower conspicuity (such as the whippen or the jack, components that in relative terms are small, visually less remarkable, and move but slightly).

Another source of challenge for learners is that the relative importance of each of the piano mechanism's various components changes over the time course of its operation. For example, the balance hammer back-check contributes its functionality only in the latter half of the mechanism's operational cycle. To understand this contribution, the learner therefore needs to direct attention to this component at just the right time (cf. Author, 2008).

If comprehension is to be fostered, learner processing of the animation should facilitate (i) extraction of information about the two interrelated causal chains that are fundamental to the piano's operation, and (ii) interlinking of that extracted information to form a coherent 
assemblage. To follow the progress of the events comprising these causal chains, learners need to begin by directing attention to the part of the display where the event chains originate. For example, particular attention needs to be given to the event unit involving the small key tail projection that contacts the whippen slightly off centre (Figure 1). However, attention capture alone could be insufficient. Learner attention must also be transferred between different event unit sites in an appropriate sequence and at a suitable rate. This requires that attention be guided appropriately through the display during the animation's time course.

On the basis of the APM, systematically cueing the piano mechanism's event units in an animation (as distinct from cueing only its entities) should help learners both with parsing, and with building micro-chunks (i.e., phases 1, 2 and 3 of the APM). This could facilitate segmentation of not only constituents of the mechanism, but their behavior as well. Most previous studies showed the positive effect of segmentation in multimedia learning (Arguel \& Jamet, 2009; Hasler, Kersten \& Sweller, 2007 and Schwan, Garsoffky \& Hesse, 2000). If the cueing also signaled spatio-temporal relations, these could then be used to build event units into larger event schemas.

\subsection{Eye movements and cue processing}

When cueing techniques are used, we need to consider what happens not only when the cue first appears, but also what happens after its initial direction of learner attention. Recent research (e.g., Authors, 2010ab, 2011; De Koning, et al., 2010; Jarodzka, Scheiter, Gerjets, \& Van Gog, 2010; Van Gog \& Scheiter, 2010) has used eye-tracking approaches to investigate the effects of cueing on multimedia learning. Most of these studies have used the total time that fixations were made on signaled information across the whole learning phase rather than more targeted measures (Hyönä, 2010). Rather than relying solely on these broad eye-tracking 
measures, more specialized time-locked analyses could "complement the global picture derived from total fixation time" (Hyönä, 2010, p. 174). In the context of research on cueing animations, time-locked analysis could be used to partition fixations into groups according to their relationships to the onset of individual cues. A number of researchers have used such time-locked analysis but typically for only short time-segments or static visualizations (see Huestegge, Skottke, Anders, Müsseler \& Debus, 2010; Jarodzka et al., 2010; Ozcelik, ArslanAri, \& Cagiltay, 2010). However, Hyönä (2010, page 175) suggested "It would be interesting to find out how fast and faithfully learners 'obey' the presented cues", not only in static depictions, but also in animations. One way to do this would be to use the cue (rather than an animation element) as the area interest.

Authors (2011) have proposed a conceptual distinction between cue obedience and cue consequence, a distinction that remains to be tested empirically. Cue obedience refers to the extent to which a cueing system is successful in the perceptual function of directing attention. We distinguished two forms of cue obedience: (i) engagement - the cue's initial capture of attention when it first appears, and (ii) loyalty - the further direction of attention to the cue beyond this initial capture. Cue consequence goes beyond the perceptual effect of cueing. Instead, it refers to the cognitive processes that occur once information signaled by a cue is internalized.

Cue engagement may be operationalized as the time to first fixation, or as the number of fixations before a first fixation is made in the target area once the cue appears in that area (i.e., cue entry). Cue loyalty may be operationalized as the relative amount of time spent viewing cued locations from the moment the cue appears (entry) until it disappears (exit). This new approach, comparing initial versus ongoing attention capture of the cue, was used in the present study to investigate the two forms of cue obedience to a dynamic cue during learning from a user controllable animation showing the upright piano mechanism. 


\subsection{Hypotheses}

Research Question: Are relational event unit cues more effective in fostering learning from a complex unfamiliar animation than conventional entity-based cues? According to the APM (Authors, 2008, 2011), the dynamic aspects of an animation can exert a powerful influence over where learner attention is directed and the processing of depicted events plays a key role in learning from complex animations. Consequently, because relational event unit cueing (i) recruits dynamic contrast to signal high relevance information more strongly and (ii) draws learner attention not just to entities but also to events and the relationships between them, it should be superior to entity cueing.

The following five hypotheses address both how different types of cueing are expected to influence attention direction during learning and the consequences of these influences for comprehension.

(i) Overall attention direction, Hypothesis 1. With regard to total viewing duration across the full learning time (as per eye movement measures), it was predicted that there would be an interaction between cueing type and salience-relevance alignment. In particular, learners in the two relational event unit cueing conditions would pay more attention to low salience, high relevance components of the piano than those in the uncued and entity cued conditions. And vice-versa, learners in the two relational event unit cueing conditions would pay less attention to high (and medium) salience and low relevance components of the piano than those in the uncued and entity cued conditions. Phase 1 of the APM concerns how selective visual attention is directed during initial parsing of the animation into individual event units. Both visuospatial and temporal properties of the displayed material determine which regions receive preferential attention from the learner. According to the APM, attention direction in an uncued animation depicting new subject matter would be largely determined by the relative 
perceptual conspicuity of the depicted event units, irrespective of their thematic relevance. Dynamic contrast plays a key role here because the human visual system privileges temporal change. Merely raising the visuospatial contrast of low salience high relevance entities (i.e., entity cueing) would not be sufficient to compete with the stronger attention directing effect of the animation's own dynamics. However, adding dynamic contrast to visuospatial contrast (as in both forms of relational event unit cueing) so that those high relevance aspects instead become strongly signaled should mean that they receive more attention than otherwise. Under these circumstances, the APM would predict those aspects to gain a higher processing priority due to the altered perceptibility profile.

(ii) Cue engagement, Hypothesis 2. It was expected that learners in the three cueing conditions would engage their attention just after the appearance of the cue. These similarities in the timing of engagement would be indicated by the time-locked data. Upon cue entry, both entity cues and relational event unit cues briefly attract attention because of a similar shortlived dynamic contrast contribution to their perceptual salience. This temporary strengthening of conspicuity can be thought of as a transition effect (Author, 2004) brought about by their sudden appearance in the display. It is only after this initial effect that relational event unit cueing adds and maintains its distinctive transformation (colour change) and translation (movement) contributions to perceptibility (Author, 2004). According to APM phase 1, both visuospatial and temporal properties contribute to conspicuity so the transition at cue entry should increase cue salience similarly in all three cued conditions.

(iii) Cue loyalty, Hypothesis 3. Obedience to the cueing was expected to extend longer beyond the initial engagement of attention in the two relational event unit cueing conditions than in the entity cueing condition, with the specific order: progressive path $>/=$ localized coordinate $>$ entity cueing. This difference should also be most marked for the first cycles of 
the animation played. Once the common initial effect of the cue entry transition has passed (see above), the fundamental differences between entity and relational event unit cueing come into play. The additional sustained dynamic contrast associated with the prolonged transformation and translation changes exhibited by the relational event unit cues raises and maintain their perceptual salience considerably over the solely visuospatial contrast of the entity cueing. On the basis of APM phase 1, we can predict that the on-going change in the perceptibility profile from the relational event unit cueing preferentially directs attention to the cued material for longer than occurs with the weaker entity cueing.

(iv) Net obedience, Hypothesis 4. Overall, cue loyalty was expected to be partial rather than total and to not persist for the whole period of a cue's exposure. According to the APM, the locations to which learners direct their attention should change over the course of animation processing. Initially, the attention directing effect of cues is needed to help learners parse the animation's continuous flux into key event units (APM phase 1). However, in order for the learner to link up these event units into progressively higher order structures, attention cannot remain exclusively on isolated cued aspects. Rather, it also needs to be shifted to neighboring aspects (APM phase 2, formation of dynamic micro chunks) and then more broadly across the display as a whole in order to link up regional activity into causal chains (APM phase 3). Without such excursions beyond the cued regions, it would not be possible for a learner to construct the network of relationships that are essential for composing a coherent mental model.

(v) Comprehension outcomes, hypothesis 5. With regard to cue consequences, it was predicted that learners in the two relational event unit cueing conditions would have superior comprehension to those in the entity and un-cued conditions, with the specific order of performance being local coordinated $>$ progressive path $>$ entity cue $=$ uncued. Both forms of 
relational event unit cueing should provide extra learning benefits because they are specifically designed to foster formation of the event units that the APM identifies as crucial to successful animation processing (see APM phase 1). However, because local coordinated cues are far more tightly targeted than progressive path cues, they should be more efficient and effective in directing learners to aspects of the animation that have particular operational significance. Further, because they do not direct attention away from elements beyond the progressive path cue track, local coordinated cues should also foster building of broader relations. Compared with progressive path cues, they should better focus learner processing and foster the broader relation-forming activities posited to occur in APM phase 2 and APM phase 3 that ultimately interconnect separate regions of the mechanism. In line with previous research (Authors, 2011), the APM would not predict strong differences between the entity cued and uncued conditions because neither of these direct attention appropriately, or specifically addresses the development of event units and their connection into higher order structures.

\section{Method}

\subsection{Participants}

84 undergraduate students of psychology ( 3 males and 81 females; mean age 19.1 years - $\underline{\mathrm{SD}}$ $=1.16)$ at a French university participated in this study for course credit. Participants were randomly assigned to each of the four conditions (with progressive path cues group, $\mathrm{n}=17$, local coordinated cues group, $\mathrm{n}=21$; entity cues group, $\mathrm{n}=21$; and uncued control group, $\mathrm{n}=$ 25). Before the experiment, participants were questioned regarding task-specific prior knowledge (Did they play the piano? Had they ever looked inside a piano? Had they ever been taught about the piano mechanism?). None of those who participated in the experiment had prior knowledge about the mechanism of a piano system. 


\subsection{Materials and Apparatus}

2.2.1. Animation. Four versions of a computer-based user-controllable piano system animation were employed. Three of these were cued while the fourth was a control version containing no cues (see Figure 2 and following descriptions).

Insert Figure 2 about here

\subsubsection{Entity Cues}

Entity cueing involved sequential signaling of entities in specific locations. Relevant components of the piano mechanism were signaled relative to the causal chain in which they were involved (Figure 2a, and also Appendix C). The basis for entity cueing was a visuospatial contrast between the target components (full colour) and the contextual components (faded same color), a signaling technique that Authors (2010b) term anti-cueing. Cueing was implemented by a smooth but rapid change in colour intensity.

\subsubsection{Progressive Path Cues}

Progressive path cues (2010a) involves the progressive application of a band of contrasting colour that spreads across related entities along the entire length of each causal chain path. One progressive path cue was applied to the causal chain for the hammer action while the other was applied to the damper's causal chain.

\subsubsection{Local Coordinated Cues}

In this approach, (Figure $2 \mathrm{c}$ and Appendix C), cueing was far more limited and tightly targeted than in progressive path cueing. Three coordinated cues were localized so that they were confined within only the most thematically relevant event areas of the piano animation. 
Each of these local cues concerned the relations between interacting sets of components: (i) key-whippen and whippen-jack, (ii) jack-butt, and (iii) spoon-damper. Short highperceptibility directional strips of colour (red and blue) were overlaid on relevant specific locations of the depicted material. In each of the cued areas, these dynamic colored cues were coordinated in time and space with operation of the mechanism. The strips cued specific events as distributed fragments of the causal chain. Upon activating the piano mechanism, the three cues started spreading in parallel at their three different locations within the animation.

\subsubsection{Animation presentation and user-control}

All versions of the animation were user-controllable, with the staging of cue presentations in the cued versions being synchronized with the animation's progression and with the learner's exercise of user control over the animation. Students activated the mechanism by clicking and moving a green arrow (representing the finger of the pianist) in a control area situated on the right side of the animation. Once activated, for each cycle of the piano they played, participants could move the arrow to play the animation forward (down) or backward (up). Changing the speed with which the arrow was moved allowed participants to play the animation at different rates so that the length of one animation cycle could be very short (e.g., 2-3 seconds) or longer (e.g., more than 10 seconds).

Cues were visible for the same amount of time across the three cued versions. There was no explanatory text. However, in all versions, pop-up labels showing piano part names were available to be viewed at any time via computer mouse roll-over. The first time a participant used the computer mouse to 'press' the piano key in the cued versions, no cues were shown. Cueing appeared only on the second and subsequent key presses, in other words, after a first complete trial cycle of the piano mechanism. This approach was designed to make it clear that the cues were not a part of the piano system but rather were an added device. 
The overall learning time allowed for studying the animation was 3 minutes. Although the duration of each cycle and number of complete cycles played could be different across learners, the total study time was constant. However, in the eye movement data analysis the time for the first cycle of the piano to be played (i.e., without cues, see above), was subtracted from the total 3 minutes study time.

\subsection{Measures}

\subsection{Comprehension test 1: the Cross Movement task}

A novel way of testing kinematic level learning was devised for this study. The cross movement task was designed to provide a more direct and appropriate measure of such learning than is possible using standard approaches such as verbal questions. It required participants to physically produce the movements of the piano mechanism's components and was a computer-based adaptation of the manipulation technique devised by Authors (2011). At the end of the learning session, a static picture of the piano mechanism in its initial state was displayed on the computer screen. As shown in Appendix A, a red cross was positioned on a part of a component. The learner was told to "use the mouse in order to move the cross to the correct final position it occupies when the key is pressed and released". In total, each participant performed the cross movement task thirty times with the cross being on a different position on every occasion. The order in which the crosses were presented (within each stage of the piano mechanism) was randomized across participants. One cross at a time was displayed. For each position of the cross, the entire movement of the mouse made by the learner was registered by the computer in real time. In order to determine kinematic accuracy, the angular direction of the movement and its amplitude were subsequently calculated and compared to the actual motion of the component as depicted in the animation. The kinematic score for each cross position was based on the angular direction (1 point) and amplitude (1 
point) of the movement (maximum was 60, see Appendix A). Scores were transformed into percentages of the total possible score.

\subsection{Comprehension test 2: Functional mental model quality}

In a second outcome measure, an open ended task was used to assess each participant's overall mental model of the piano mechanism. Participants were asked to "write as much as you can about what happens (events) with all entities (components) of the system when someone presses the key down and then releases it". In order to avoid difficulties related to the recall of technical names of the components, participants were also given a sheet of paper upon which labeled pictures of the piano components were shown in random positions. The functional mental model quality scoring guide was based on the 15 micro-steps constituting the three main stages of a piano mechanism's functioning (see Appendix B). Each correct micro-step (entities plus their behaviors) was awarded one point if fully and accurately reported or half a point if reported only in part (maximum: 15). Participants' answers were scored by two independent raters, with inter-rater agreement, chance corrected Cohen's kappa, being .93. Scores were transformed into percentages of the total possible score.

\subsubsection{Eye tracking equipment and data analysis.}

Eye movements were recorded with a $50 \mathrm{~Hz}$ Tobii, 1750 binocular corneal reflectance and pupil centre eye tracker (Tobii-Studio software). Instead of fixation duration, a dwell time measure was used (Hyönä, 2010; Hyönä, Radach \& Deubel, 2003; Jarodzka, et al., 2010). Dwell times derived from raw data were based on Areas of Interest (AOIs), each of which corresponded to a functional part of the piano system (Figure 2d). Two types of analysis were 
carried out on the eye tracking data: (i) the total viewing duration in different AOIs summed across the overall learning time was used to measure to what extent the different types of cueing attracted learners' attention to relevant components of the piano mechanism (hypothesis 1 above). (ii) Time locked measures across cued time-segments of the animation cycles played by each participant were used with dynamic AOIs to determine cue engagement and cue loyalty (hypotheses, 2, 3 and 4).

For total viewing duration across time on task, AOIs for the same nine piano components were used in all four conditions (Fig. 2d). Each of these AOIs was defined with sufficient scope to include not only cues in the cued conditions, but also the boundaries of the particular piano part's entire movement during its operational cycle (so event's areas were included in each of the AOIs). A further Null AOI category was also established that covered all Not-onAOI areas such as the region containing the arrow for controlling the animation (Fig. $2 \mathrm{~d}$ ) and the spaces between the other nine AOIs. Total viewing durations in each of the ten AOIs across the time on task were determined. In order to test hypothesis 1 concerning the effectiveness of cueing in directing attention to low salience, high relevance components of the piano mechanism, the nine component AOIs were assigned to three categories with respect to their thematic relevance and perceptual salience (Schnotz \& Lowe, 2008). The first category contains components with relatively low perceptual salience (LS) but higher order thematic relevance (HR), that is, the end of the key, the whippen, the jack, the hammer-butt and the spoon (LS/HR). In the second category are components with medium perceptual salience (MS) and lower thematic relevance, that is, the damper, and the balance-back-check system (MS/LR). The third category involves components with high perceptual salience (HS) and also high thematic relevance (HR), that is the hammer and the hammer move (plus string) area, (HS/HR). The main overall effect of the different cueing conditions on the direction of 
attention on the different piano components was characterized using the total viewing duration in each of the LS/HR, MS/LR, and HS/HR AOI groups. To avoid bias, raw data were normalized by converting them to percentages of the time on task spent for the different AOI groups. Differences in AOI sizes (areas) were also checked. It should be noted that even if the number of components per AOI within a group was not the same across the three groups, the overall areas of each of the three groups of AOIs were similar.

For the time locked analysis, the full set of eye tracking data collected for each participant was partitioned into dynamic time-segments that covered particular cueing instances. The dynamic AOIs assigned for this time locked analysis depended on whether the component under consideration was cued at a given point in time or not. This meant that a particular component of the display (e.g., the whippen) was assigned to 'AOI-cued' from the moment its cue arrived in the component's AOI (entry) until the cue's exit from that AOI. We use the term excluded to refer collectively to areas containing components that were either never cued in a particular condition (e.g., the damper in the local coordinated condition), or that were not the subject of active cueing action during the period under consideration (e.g., the jack in the entity condition while the whippen is being cued).

Because the animation was user-controllable, the temporal cut-offs were not identical across individuals but were determined for every subject, according to common boundaries based on the piano mechanism's operational cycle. Appendix C illustrates these boundaries using the Strike stage of the piano's operation as an example. The same technique was also used for the Rebound and Reset stages.

For each complete cycle of the piano played, the time locked data for each time segment were accumulated across all inspections of that segment made by an individual participant. For the purposes of analyzing participants' gazes with respect to each time segment, the piano 
mechanism areas of the display was then divided into two dynamic AOIs: (i) the area in which the cue was dynamically applied (from entry of the cue into the AOI until its exit from that AOI), and (ii) the excluded area, (AOIs where cueing was either never applied or was not currently active). The Null AOI area (all Not-on-AOI areas: the region containing the arrow for controlling the animation and the spaces between the nine AOIs) was also taken into account for checking purposes.

Two types of dependent measures were used for each segment. The first of these was concerned with cue loyalty and measured the viewing durations (dwell time raw data) in the cued area versus the excluded area throughout the whole segment. Time on task was not equal to the overall study time constraint of 3 minutes because the first trial without cues was excluded. In order to normalize all data with time on task, percentages in the cued and excluded area were used. The second measure was concerned with cue engagement and measured how many fixations it took after the onset of the cue presentation before fixations 'arrived' in the cued area and in the excluded AOIs. If only strict raw data were to be taken into account for this second measure, the result may include very short glances $(20 \mathrm{~ms})$ on an AOI that would not be meaningful. To avoid this problem, we therefore set a lower threshold for time spent on an AOI of 100ms.

\subsection{Procedure}

The experiment was run on an individual basis with participants seated at the computer. After being asked about their possible task-specific prior knowledge, they were instructed to study the animation in order to understand how the piano mechanism works (that is to say, both the entities and their behavior) in preparation for a subsequent comprehension test. The eye tracker was calibrated for each participant immediately prior to the session. After having 
completed their 3 minutes study of the animation, participants undertook the comprehension tests.

\section{Results}

\subsection{Eye tracking data}

Analyses were performed with "Statistica 10" software, which computes automatically both ANOVA/MANOVA tests (General Linear Models of ANOVA or MANOVA depending on the number of levels of the within group factor).

\subsubsection{Overall fixation duration in groups of AOIs}

Table 1 presents the mean total viewing durations (during time on task), raw data and percentages (normalized for time on task), in the three Salience-Relevance alignment (S-R) AOI groups (LS/HR, MS/LR, HS/HR) and the Null AOI for each cueing condition.

Insert Table 1 about here

To check for comparability of time on task and Null AOI time across conditions, two single factor ANOVAs were performed), each with type of cueing condition as between factor. These analyses showed no significant effect for either time on task, $F(3,80)=1.13, p=.34$, $\eta_{p}^{2}=.04$ or Null AOI time, $F(3,80)=1.1, p=.36, \eta_{p}^{2}=.04$

Following this check, a repeated measure ANOVA with type of cueing condition as between factor and Salience-Relevance alignment (S-R) AOI groups as within subject factor (single level) was performed on the percentage of time spent in the three S-R AOI groups. 
This analysis showed that for the three S-R AOI groups taken together, there was no effect of the cueing condition on total viewing duration, $F(3,80)=1.1, p=.34 \eta_{p}{ }^{2}=.04$. The analysis revealed a main effect of S-R AOI group, $F(2,160)=158.61, p<.0001, \eta_{p}{ }^{2}=.66$. Viewing duration was lower in the HS/HR group of AOIs than in the two other groups of AOIs. As predicted by Hypothesis 1, a significant interaction was found between the type of cueing and S-R AOI group $F(6,160)=23.58, p<.0001, \eta_{p}{ }^{2}=.47$.

The specific prediction of Hypothesis 1 was that, compared to the uncued and entity cueing conditions, the two relational event unit cueing conditions would result in learners paying more attention to low salience, high relevance components of the piano and vice versa, learners in the two relational event unit cueing conditions would pay less attention to high, or medium salience and low relevance components of the piano than those in the uncued and entity cued conditions. To examine this prediction, univariate ANOVAs were performed for each S-R AOI group. The LS/HR group ANOVA revealed an overall effect of the type of cueing $F(3,80)=30.86, p<.0001, \eta_{p}{ }^{2}=.54$, with (i) progressive path and local coordinated conditions $>$ entity and uncued conditions, $F(1,80)=40.70, p<.0001$, (ii) local coordinated condition $>$ progressive path condition, $F(1,80)=35.30, p<.0001$, and (iii) progressive path condition $>$ entity and uncued conditions, $F(1,80)=4.23, p=.043$. The HS/HR group ANOVA also revealed an overall effect of cueing type, $F(3,80)=4.38, p<.01, \eta_{p}^{2}=.14$, with (i) progressive path condition $>$ local coordinated condition, $F(1,80)=12.90, p<.01$, (ii) progressive path $>$ entity and uncued conditions, $F(1,80)=4.27, p<.05$, and (iii) entity and uncued conditions $>$ local coordinated, $F(1,80)=5.09, p<.03$. Finally, the MS/LR group ANOVA again revealed an overall effect of cueing type $F(3,80)=17.82, p<.001, \eta_{p}{ }^{2}$ $=.40$, with (i) entity cue and uncued conditions > progressive path and local coordinated conditions, $F(1,80)=40.99, p<.0001$, and (ii) progressive path $>$ local coordinated, $F(1$, 
$80)=5.99, p<.02)$. However, there was no difference between the entity and uncued conditions, $F(1,80)=0.81, p=.37$.

\subsubsection{Time locked measures in dynamic AOIs: cue obedience, engagement and loyalty}

Table 2 shows time locked analysis results. To test hypotheses 2 and 3 about cue obedience, engagement and loyalty, analyses were performed for both (i) an accumulation of all the cycles played by the learner (Accumulated, lower part of table 2) and for (ii) just the first two cycles played (Initial, upper part of table 2) in which cues were present. The uncued condition was not included in the analysis because such comparison without cues was not considered meaningful.

Insert table 2 about here

Two preliminary one factor ANOVAs for the Null AOI time were firstly performed, one for the viewing duration and another for the arrival lag. With respect to viewing duration, there was no significant difference across the cueing conditions for either the accumulated cycles $F$ $(2,56)=2.04, p=.14, \eta_{p}{ }^{2}=.07$ or the initial cycles $F(2,56)=0.23, p=.79, \eta_{p}{ }^{2}=.008$. Further, there was no significant difference with respect to arrival lag for either the accumulated cycles $\mathrm{F}(2,56)=0.01, \mathrm{p}=.98, \eta_{p}{ }^{2}=.0005$ or the initial cycles, $F(2,56)=0.5, p$ $=.94, \eta_{p}^{2}=.001$.

Analyses with cueing type as between factor and cued versus excluded areas as within factor (single level) were performed on the time-locked data. For the engagement measure (mean number of fixations before arrival of attention in the cued area and in the excluded area), a repeated measure ANOVA with cueing type as between group factor, and AOIs (cued vs. 
excluded) as within factors was performed for both the accumulated and initial cycles measures.

The analysis for the accumulated measure revealed an effect of AOIs, $F(1,56)=14.55, p<$ $.001, \eta_{p}{ }^{2}=.21$ which indicated that upon cue entry, learners arrived sooner in the cued AOI than in excluded AOIs. No significant overall effect was found for the cueing condition $F$ (2, 56) $=3.14, p=.051, \eta_{p}{ }^{2}=.10$. The interaction between AOIs and cueing condition was significant $F(2,56)=27.60, p<.001, \eta_{p}{ }^{2}=.49$. This interaction showed learners arrived sooner on the cued AOIs than on the excluded AOIs in the progressive path condition $F(1$, $56)=8.56, p<.005$, and in the local coordinated condition, $F(1,56)=50.99, p<.0001$; but arrived later on the cued AOI than on the excluded AOI in the entity condition $F(1,56)=$ 11.16, $p<.005$. As would be expected, the Null AOI (containing the user-control area) was the first visited.

The analysis for the initial cycles showed the same pattern of result with (i) a significant effect of AOIs $, F(1,56)=15.93, p<.0001, \eta_{p}{ }^{2}=.22$, (ii) no overall effect of cueing conditions, $F(2,56)=1.30, p=.28, \eta_{p}{ }^{2}=.04$, and (iii) an interaction between AOIs (cued vs excluded) and cueing conditions, $F(2,56)=4.02, p=.023, \eta_{p}{ }^{2}=.12$. Learners arrived sooner in the cued AOIs than in the excluded AOIs in the progressive path condition $F(1,56)=$ $12.79, \mathrm{p}<.005$, and in the local coordinated condition, $F(1,56)=9.53, \mathrm{p}<.004$; but arrived approximatively at the same time in the cued or excluded AOIs in the entity condition $F$ (1, 56) $=.006, p=.93$.

For the loyalty measure (i.e., the viewing duration in the cued area with respect to time segments), repeated measures ANOVAs with cueing type as between group factor and cued/excluded AOIs as within factor (single level) were performed for both the accumulated and initial cycles measures. 
The analysis for accumulated cycles showed an effect of AOIs, $F(1,56)=19.22, p<.0001$, $\eta_{p}{ }^{2}=.25$, which revealed that learners spent more time on cued areas than on excluded areas. No overall effect was found across the cueing conditions, $F(2,56)=2.04, p=.14, \eta_{p}{ }^{2}=.06$. However, the interaction between the type of cueing and the AOIs was significant, $F(2,56)=$ $5.66, p=.005, \eta_{p}{ }^{2}=.17$. In the progressive path condition, learners spent significantly more time on cued AOIs than on excluded AOIs, $F(1,56)=22.95, p<.0001$. However, this was not the case for the other conditions: local coordinated, $F(1,56)=0.58, p=.44$; entity, $F(1$, 56) $=2.50, p=.12$.

The analysis for the initial cycles indicated a different pattern of results. Learners spent more time on the cued area than on the excluded areas, $F(1,56)=35.50, p<.0001, \eta_{p}{ }^{2}=.39$. No overall effect was found for cueing conditions, $F(2,56)=0.23, p=0.79, \eta_{p}{ }^{2}=.008$. The interaction between the type of cueing and the AOIs was significant, $F(2,56)=7.11, p<$ $.002, \eta_{p}{ }^{2}=.20$. For the two relational event unit cues, participants spent more time on cued AOIs than on excluded AOIs: progressive path, $F(1,56)=18.91, p<.0001$; local coordinated, $F(1,56)=29.74, p<.0001$. However, this was not the case for entity cues $F(1$, 56) $=0.23, p=.62$.

Finally, regarding hypothesis 4 about net obedience to cues, the results presented above show that while the cue was present, participants' attention was not exclusively devoted to the cued areas - they also attended to the excluded areas. This supports the prediction made on the basis of the APM that in order to connect the individual event units identified in APM phase 1 via relational processing during APM phases 2 and 3, cue obedience should be partial rather than total. 


\subsection{Comprehension measures}

Table 3 shows the variation of comprehension performance (raw scores and percentages) with respect to (i) kinematics (cross-movement task) and (ii) functional mental model quality (written task) as a function of cue condition.

Insert Table 3 about here

To test hypothesis 5 which predicted the specific order of performance being local coordinated $>$ progressive path $>$ entity cue $=$ uncued; two single factor ANOVAs, with cueing condition as between subject factor and comprehension scores as dependent measures, were used, one for the cross movement kinematics task measure, and the other for the written mental model task measure.

With the cross-movement task, there was a significant effect for cueing condition $F(3,80)=$ 24.29, $p<.001, \eta_{p}{ }^{2}=.48$. With respect to hypothesis 5 , planned comparisons indicated that learners in the progressive path and local coordinated cue conditions outperformed learners in the entity and uncued conditions $F(1,80)=66.86, p<.001, \eta_{p}{ }^{2}=.44$. However, there was no difference between the local coordinated and progressive path cue conditions $F(1,80)=1.24$, $p=.26, \eta_{p}{ }^{2}=.03$. The difference between the entity cue and uncued conditions was marginal $F(1,80)=3.85, p=.053, \eta_{p}{ }^{2}=.06$. In summary, progressive $=$ local $>$ entity $\geq$ uncued.

There was also a significant effect for cueing condition with the written mental model measure, $F(3,80)=8.43, p<.001, \eta_{p}{ }^{2}=.24$. Planned comparisons indicated that learners in the progressive and local cue conditions outperformed learners in the entity and uncued conditions, $F(1,80)=23.15, p<.0001, \eta_{p}{ }^{2}=.21$. However, the difference between the local 
and progressive conditions was not significant $F(1,80)=2.65, p=.11, \eta_{p}{ }^{2}=.06$. There was also no significant difference between the entity and uncued conditions, $F(1,80)=.79, p=$ $.37, \eta_{p}^{2}=.01$. In summary, progressive $=$ local $>$ entity $=$ uncued.

As a final check on the comprehension results, user-control of the animation was examined. The number of complete operational cycles of the piano played by participants within each experimental condition during the total time on task was analyzed. Differences were found as follows: progressive path $(M=7.40, S D=3.6)<$ local coordinated $(M=10.08, S D=6.86)<$ Entity $(M=11.33, S D=6.35)<$ uncued $(\mathrm{M}=11.80, \mathrm{SD}=7.65)$. However, a single factor ANOVA performed on the number of cycles played, with cueing condition as between factor, did not show a significant overall effect $F(3,80)=1.58, p=.19, \eta_{p}{ }^{2}=.05$.

\subsection{Eye tracking data and comprehension}

A multiple regression to examine the effect of viewing duration for the three $\mathrm{S}-\mathrm{R}$ AOI groups (LS/HR; MS/LR; HS/HR) on kinematic comprehension score was performed. This analysis was limited to the broad overall effect of the AOI groups factor, because more loyalty as well as faster engagement do not mean automatically more comprehension. Also, this analysis was restricted to the cross movement task which represents a more direct measure of spatiotemporal cognitive representations than the written measure involving an additional wording activity. The results indicated a significant effect, $R^{2}=.197, F(3,80)=6.57, p=.0005$, with the LS/HR group of AOIs explaining most of the variance, $\beta=.43, t(80)=3.14, p=.002$ compared to the two other groups of AOIs, respectively, for MS/LR, $\beta=-.08, t(80)=-.68, p$ $=.49$, and for HS/HR, $\beta=.10, t(80)=.87, p=.38$. 


\section{Discussion and conclusion}

The types of conventional visual cues that are effective with static representations have met with limited success when used in animations, particularly those that depict complex unfamiliar subject matter (de Koning et al., 2009, 2010). This difference has been attributed to the distinctive processing challenges that animated graphics pose to learners because of their transient character (Spanjers \& al., 2010). The goal of this study was to examine the learning effectiveness of dynamic relational event cueing techniques compared to conventional cueing.

Two of the three cued conditions employed were different types of dynamic relational event unit cueing designed on the basis of the Animation Processing Model (APM, Authors 2008): (i) progressive path cues and (ii) local coordinated cues. The design of these cues was intended to (i) strengthen attention direction within the context of an animation by their incorporation of dynamic contrast, and (ii) explicitly signal key events and relationships. It was expected that these design features would support more effective parsing, building of dynamic micro chunks, and elaboration of broader regional structures (APM phases 1, 2, and 3). The remaining conventionally cued condition signaled relevant entities across the set of depicted events but did not cue the events or relations themselves. There was also an uncued control condition.

As predicted by Hypothesis 1, eye tracking data indicated that learners in the two relational event unit cueing conditions paid more attention overall to low salience, high relevance components of the display than those in the uncued and entity cued conditions. This is consistent with the APM-based expectation that combining dynamic contrast with visuospatial contrast would alter the perceptibility profile of the display to strengthen the signaling of high relevance aspects sufficiently for them to receive due attention from learners. More precisely with respect to all results, in the high salience, high relevance case 
(HS/HR),the combined effect of (a) the natural cueing from the HS components' movements (e.g. the Hammer) and (b) the applied cueing (i.e., relational) produced stronger signaling than the natural cueing alone. In the medium salience case (MS/LR), the applied relational cues, in one part of the mechanism, outcompeted the moderately salient natural cues in a different part of the mechanism.

Consistent with Hypothesis 2, the initial engagement of attention did not differ between the entity cued and relational event unit cued conditions. The time-locked eye tracking measure showed that in all three conditions, learners first engaged their attention just after the cue appeared. Subsequently, as predicted by Hypothesis 3, obedience to cueing extended longer for the two relational event cueing conditions than for the entity cued condition. This distinction between cue engagement and cue loyalty can be partly attributed to the on-going change in perceptibility profile that is present with relational event unit cueing but not with entity cueing. However, the effect concerned both progressive path cueing and localized coordinate cueing for the initial cycles but only progressive path cueing and not localized coordinate cueing for the accumulated cycles measure.

Despite cue loyalty being greater for relational event cueing than for entity cueing, as predicted by Hypothesis 4, loyalty was partial rather than total. Once an animation has been parsed to identify key event units, the learner's task is then to link these into progressively higher order dynamic structures and so build a coherent mental model of the referent content.

The Hypothesis 5 prediction that comprehension would be superior for the two relational event unit cueing conditions was confirmed. This is attributed to the net effect of (a) greater learner attention overall to low salience, high relevance aspects throughout the piano 
mechanism's operational cycle (b) coupled with the progress of cueing through successive attention targets, that enables their interrelation into coherent higher order structures. However, contrary to expectations, no comprehension difference was found between the local coordinated and progressive path conditions. As indicated above, the extent to which cues are obeyed may not be only a matter of their bottom-up influence. In the localized coordinated cueing condition, short high-perceptibility directional strips of colour cued specific events as distributed (spatially separated) fragments of the causal chain. Compared with progressive path cues, they should better focus learner processing and foster the broader relation-forming activities posited to occur in APM phase 2 and APM phase 3 that ultimately interconnect separate regions of the mechanism. However, this distributed cueing method could result in less bottom-up guidance than the progressive path cues. Consequently, learners can of course exert considerable top-down control over how much regard they give to the provided signals. Cues should therefore be regarded as providing various processing opportunities, not limited processing prescriptions. To benefit from such opportunities, learners need to adopt a relationship-oriented and flexible strategy to their processing of the available information (Kühl, Scheiter, Gerjets \& Gemballa, 2011).

A possible limitation of this study is the use of anti-cueing for signaling in the conventional entity cueing condition but the use of ribbons of colour in both relational event unit cueing conditions. Technically speaking, a difference therefore exists not only in what is cued, but also in how this is cued (i.e., lowered colour intensity versus superimposition of a contrasting colour). It is therefore possible that the difference found between the relational event unit cueing and the entity cueing is not due purely to which of these types of cueing was used. Rather, it may also be partly due to colour differences. Using the same animation, Authors (2011) found that anti-cues could indeed be less effective than colour cues and suggested that 
this was because anti-cueing may indicate to learners that they can neglect the faded (nonsignaled) parts of the display. This in turn could lead to different distributions of learner attention for anti-cued versus colour cued approaches. However, the difference that Authors found between these two cueing approaches was evident for only the first inspection of the animation. When the whole viewing time was considered, this effect disappeared. Moreover, in another recent study, with the same piano mechanism, Authors (2012) found no difference between an uncued condition and an entity cueing condition using colored temporal tokens.

In the present study, the eye movement data suggest that the significant difference found between relational event cueing and entity cueing is due in part at least to their distinctive effects on learner processing. Further, if the anti-cueing caused participants in this study to neglect faded (non-cued) parts, comprehension performances should have been inferior to that of those in the un-cued control condition (in which all entities are equally colored). However, this was not the case, with performance in the anti-cued condition actually being marginally superior to that in the un-cued condition. Nevertheless, explicit comparison of relational event unit cueing and superimposed colour cueing should be undertaken too.

In sum, this study suggests the Animation Processing Model provides a principled basis for designing interventions that can increase the instructional effectiveness of educational animations.

\section{References}

Amadieu, F., Mariné, C., \& Laimay, C. (2011). The attention guiding effect and cognitive load in the comprehension of animations. Computers in Human Behavior, 27, 36-40. doi:10.1016/j.chb.2010.05.009.

Arguel, A., \& Jamet, E. (2009). Using video and static pictures to improve learning of procedural contents. Computers in Human Behavior, 25, 354-359. doi:10.1016/j.chb.2008.12.014.

Ayres, P., \& Paas, F. (2007a). Can the cognitive load approach make instructional animations more effective? Applied Cognitive Psychology, 21, 811-820. doi:10.1002/acp.1343.

Ayres, P., \& Paas, F. (2007b). Making instructional animations more effective: a cognitive load approach. Applied Cognitive Psychology, 21, 695-700. doi:10.1002/acp.1351. 
Authors. (1999).

Authors. (2003)

Authors. (2004).

Authors. (2008).

Authors. (2010a).

Authors. (2010b).

Authors. (2011).

Authors. (2012).

Barrouillet, P., Bernardin, S., Portrat, S., Vergauwe, E., \& Camos, V. (2007). Time and cognitive load in working memory. Journal of Experimental Psychology: Learning, Memory, and Cognition, 33, 570-585. doi: 10. 1037/0278-7393.33.3.570.

Betrancourt, M. (2005). The animation and interactivity principles in multimedia learning. In R. Mayer (Ed.), The Cambridge handbook of multimedia learning (pp. 287-296). Cambridge, UK: Cambridge University Press.

De Koning, B. B., Tabbers, H. K., Rikers, R. M. J. P., \& Paas, F. (2007). Attention cueing as a mean to enhance learning from an animation. Applied Cognitive Psychology, 21, 731746. doi:10.1002/acp.1346.

De Koning, B. B., Tabbers, H. K., Rikers, R. M. J. P., \& Paas, F. (2009). Towards a framework for attention cueing in instructional animations: Guidelines for research and design. Educational Psychology Review, 21, 113-140. doi 10.1007/s10648-009-9098-7.

De Koning, B. B., Tabbers, H. K., Rikers, R. M. J. P, \& Paas, F. (2010). Attention guidance in learning from a complex animation: Seeing is understanding? Learning and Instruction, 20, 111-122. doi:10.1016/j.learninstruc.2009.02.010.

Fischer, S., \& Schwan, S. (2008, July). Temporal manipulations for animation design: Presentation speed outperforms cueing. Paper presented at the XXIX International Congress of Psychology (ICP). Berlin.

Fischer, S., Lowe, R. K., \& Schwan, S. (2008). Effects of presentation speed of a dynamic visualization on the understanding of a mechanical system. Applied Cognitive Psychology, 22, 1126-1141. doi:10.1002/acp.1426.

Hasler, B. S., Kersten, B., \& Sweller, J. (2007). Learner control, cognitive load and instructional animation. Applied Cognitive Psychology, 21, 713-729. doi: 10.1002/acp.1345.

Höffler, T. N., \& Leutner, D. (2007). Instructional animation versus static pictures: a metaanalysis. Learning and Instruction,17, 722-738. doi:10.1016/j.learninstruc.2007.09.013.

Huestegge, L., Skottke, E.-M., Anders, S., Müsseler, J. \& Debus, G. (2010). The development of hazard perception: Dissociation of visual orientation and hazard processing. Transportation Research, Part F: Traffic Psychology and Behaviour 13(1), 1-8.

Huk, T., Steinke, M., \& Floto, C. (2003). Helping teachers developing computer animations for improving learning in science education. Albuquerque, USA: Proceedings of the Society for Information Technology and Teacher Education.

Hyönä, J., Radach, R., \& Deubel, H. (2003). The mind's eye: Cognitive and applied aspects of eye movement research. Amsterdam: Elsevier.

Hyönä, J. (2010). The use of eye movements in the study of multimedia learning. Learning and Instruction, 20, 172-176. doi:10.1016/j.learninstruc.2009.02.013.

Jamet, E., Gavota, M., \& Quaireau, C. (2008). Attention guiding in multimedia learning. Learning and Instruction, 18, 135-145. doi:10.1016/j.learninstruc.2007.01.011.

Jarodzka, H., Scheiter, K., Gerjets, P., \& Van Gog, T. (2010). In the eyes of the beholder: How experts and novices interpret dynamic stimuli. Learning and Instruction, 20, 146154. doi:10.1016/j.learninstruc.2009.02.019. 
Kühl, T., Scheiter, K., Gerjets, P., \& Gemballa, S. (2011). Can differences in learning strategies explain the benefits of learning from static and dynamic visualizations? Computers and Education, 56, 176-187. doi:10.1016/j.compedu.2010.08.008.

Kurby, A., \& Zacks, J. (2007). Segmentation in the perception and memory of events. Trends in Cognitive Science, 12, 72-79. doi:10.1016/j.tics.2007.11.004.

Kriz, S., \& Hegarty, M. (2007). Top-down and bottom-up influences on learning from animations. International Journal of Human-Computer Studies, 65, 911-930. doi:10.1016/j.ijhcs.2007.06.005.

Lin, L., \& Atkinson, R. (2011). Using animations and visual cueing to support learning of scientific concepts and processes. Computers and Education, 56, 650-658. doi:10.1016/j.compedu.2010.10.007.

Mautone, P. D., \& Mayer, R. E. (2001). Signaling as a cognitive guide in multimedia learning. Journal of Educational Psychology, 93, 377-389. doi:10.1037/00220663.93.2.377.

Moreno, R. (2007). Optimizing learning from animations by minimizing cognitive load: Cognitive and affective consequences of signaling and segmentation methods. Applied Cognitive Psychology, 21, 765-781. doi:10.1002/acp.1348.

Narayanan, N. H., \& Hegarty, M. (1998). On designing comprehensible interactive hypermedia manuals. International Journal of Human-Computer Studies, 48(2), 267301.

Ploetzner, R, \& Lowe, R.K. (2012). A systematic characterization of expository animations. Computers in Human Behavior, 28(3), 781-794. doi:10.1016/j.chb.2011.12.001.

Ozcelik, E., Arslan-Ari, I., \& Cagiltay, K. (2010). Why does signaling enhance multimedia learning? Evidence from eye tracking. Computer in Human Behavior, 26, 110-117. doi:10.1016/j.chb.2009.09.001.

Schnotz, W., \& Lowe, R.K. (2008). A unified view of learning from animated and static graphics. In R.K. Lowe \& W. Schnotz (Eds), Learning with animation: Research Implications for Design. New York: Cambridge University Press.

Schwan, S., Garsoffky, B., \& Hesse, F. W. (2000). Do film cuts facilitate the perceptual and cognitive organization of activity sequences? Memory \& Cognition, 28, 214-223.

Spanjers, I.A.E, Van Gog, T., \& Van Merrienboer, J.J.G, (2010). A theoretical analysis of how segmentation of dynamic visualizations optimizes students' learning. Educational Psychology Review, 411-423, doi: 10.1007/s10648-010-9135-6.

Van Gog, T. \& Scheiter, K. (2010). Eye tracking as a tool to study and enhance multimedia learning. Learning and Instruction, 20, 95-99. doi:10.1016/j.learninstruc.2009.02.009.

Wolf, M.W. \& Horowitz, T.S. (2004). What attributes guide the deployment of visual attention and how they do it? Neuroscience, 5, 1-7.

Wouters, P., Paas, F., \& Van Merriënboer, J. J. G. (2008). How to optimize learning from animated models: A review of guidelines based on cognitive load. Review of Educational Research, 78, 645-675. doi:10.3102/0034654308320320. 


\section{Acknowledgments}

This research was a part of larger international project "COGNIMAG" funded by the French National Research Agency, A. N. R (2007-2010). The authors thank Stéphane Argon for his invaluable assistance in implementing the interactive piano animation used in the studies. 
Tables

Table 1.

Mean viewing durations (dwell time) in second (with SD) and percentage (with SD) in the three groups of AOIs*, by cueing condition**

\begin{tabular}{|c|c|c|c|c|}
\hline & \multicolumn{4}{|l|}{ Cueing condition } \\
\hline AOI group & Uncued & Entity & Local & Progressive \\
\hline LS/HR & $46.89(12.97)$ & $45.66(13.12)$ & $84.67(24.26)$ & $55.66(11.23)$ \\
\hline Key-Whippen-Jack- & $29.29 \%(7.71)$ & $28.80 \%(7.81)$ & $52.54 \%(13.50)$ & $33.55 \%(7.42)$ \\
\hline \multicolumn{5}{|l|}{ Butt-Spoon } \\
\hline HS/ HR & $18.52(7.51)$ & $18.43(7.25)$ & $12.82(16.71)$ & $26.14(9.53)$ \\
\hline Hammer & $11.56 \%(4.46)$ & $11.68 \%(4.42)$ & $7.90 \%(9.61)$ & $15.58 \%(5.44)$ \\
\hline \multicolumn{5}{|l|}{ Hammer-move } \\
\hline MS/LR & $63.32(14.92)$ & $66.91(15.65)$ & $39.12(16.59)$ & $52.93(13.92)$ \\
\hline Balance-back- & $39.67 \%$ (9.27) & $42.08 \%(8.27)$ & $24.36 \%(9.96)$ & $31.69 \%(8.04)$ \\
\hline \multicolumn{5}{|l|}{ check-Damper } \\
\hline \multirow{2}{*}{$\begin{array}{l}\text { Null AOIs } \\
\text { (with user control }\end{array}$} & $31.25(19.84)$ & $27.34(7.80)$ & $24.70(12.62)$ & $31.90(13.53)$ \\
\hline & $19.47 \%(12.21)$ & $17.44 \%(5.14)$ & $15.19 \%(7.50)$ & $19.17 \%(8.20)$ \\
\hline Time on task** & $160.01(12.06)$ & 158.35 (15.73) & $161.31(16.71)$ & $166.64(7.63)$ \\
\hline (3'minus cycle 0 ) & & & & \\
\hline
\end{tabular}

* derived from the original nine AOIs

** In the different cueing type conditions, the time of the first cycle of the piano played (cycle 0 , first trial without cues), was subtracted from the total 3 minutes study time. 
Table 2.

Comparison of arrival lag* and total dwell time duration (\%) by cueing condition, for the two initial cycles (with cues) and for all accumulated cued time-segments in the cued AOI, in the excluded AOI and in the Null AOIs.

\begin{tabular}{|c|c|c|c|c|c|c|c|}
\hline & & \multicolumn{2}{|c|}{ Progressive path cue } & \multicolumn{2}{|c|}{ Localized coord.cue } & \multicolumn{2}{|c|}{ Entity cue } \\
\hline & & $\begin{array}{l}\text { Arrival } \\
\text { lag* }\end{array}$ & $\begin{array}{l}\text { Viewing } \\
\text { Duration }\end{array}$ & $\begin{array}{l}\text { Arrival } \\
\text { lag }\end{array}$ & $\begin{array}{l}\text { viewing } \\
\text { Duration }\end{array}$ & $\begin{array}{l}\text { Arrival } \\
\text { lag }\end{array}$ & $\begin{array}{l}\text { viewing } \\
\text { Duration }\end{array}$ \\
\hline \multirow[t]{3}{*}{$\begin{array}{l}\text { Cycles } \\
1 \text { and } 2\end{array}$} & $\begin{array}{l}\text { Cued } \\
\text { AOI }\end{array}$ & $\begin{array}{c}6.52 \\
(1.43)\end{array}$ & $\begin{array}{c}54.87 \\
(10.68)\end{array}$ & $\begin{array}{c}6.24 \\
(1.53)\end{array}$ & $\begin{array}{c}54.03 \\
(17.68)\end{array}$ & $\begin{array}{c}6.38 \\
(1.86)\end{array}$ & $\begin{array}{l}42.38 \\
(8.56)\end{array}$ \\
\hline & $\begin{array}{l}\text { Excluded } \\
\text { AOI }\end{array}$ & $\begin{array}{c}8.27 \\
(2.48)\end{array}$ & $\begin{array}{c}28.65 \\
(10.07)\end{array}$ & $\begin{array}{c}7.46 \\
(2.43)\end{array}$ & $\begin{array}{c}27.49 \\
(13.55)\end{array}$ & $\begin{array}{c}6.41 \\
(2.37)\end{array}$ & $\begin{array}{c}39.89 \\
(10.07)\end{array}$ \\
\hline & $\begin{array}{l}\text { Null AOIs } \\
\text { (with user } \\
\text { control area) }\end{array}$ & $\begin{array}{c}6.59 \\
(1.66)\end{array}$ & $\begin{array}{l}16.48 \\
(7.88)\end{array}$ & $\begin{array}{c}6.44 \\
(3.32)\end{array}$ & $\begin{array}{l}18.47 \\
(8.33)\end{array}$ & $\begin{array}{c}6.31 \\
(1.87)\end{array}$ & $\begin{array}{l}17.73 \\
(9.82)\end{array}$ \\
\hline \multirow{3}{*}{$\begin{array}{l}\text { All } \\
\text { accumulated } \\
\text { cycles }\end{array}$} & $\begin{array}{l}\text { Cued } \\
\text { AOI }\end{array}$ & $\begin{array}{c}4.28 \\
(0.92)\end{array}$ & $\begin{array}{l}53.81 \\
(9.27)\end{array}$ & $\begin{array}{c}4.93 \\
(1.15)\end{array}$ & $\begin{array}{c}45.40 \\
(14.23)\end{array}$ & $\begin{array}{c}6.01 \\
(1.17)\end{array}$ & $\begin{array}{l}46.01 \\
(8.33)\end{array}$ \\
\hline & $\begin{array}{l}\text { Excluded } \\
\text { AOI }\end{array}$ & $\begin{array}{c}5.42 \\
(1.77)\end{array}$ & $\begin{array}{l}29.30 \\
(9.79)\end{array}$ & $\begin{array}{c}7.17 \\
(2.21)\end{array}$ & $\begin{array}{c}42.24 \\
(10.92)\end{array}$ & $\begin{array}{c}4.91 \\
(1.53)\end{array}$ & $\begin{array}{l}39.18 \\
(8.07)\end{array}$ \\
\hline & $\begin{array}{l}\text { Null AOIs } \\
\text { (with user } \\
\text { control area) }\end{array}$ & $\begin{array}{c}4.00 \\
(1.15)\end{array}$ & $\begin{array}{l}16.88 \\
(8.10)\end{array}$ & $\begin{array}{l}4.10 \\
(2.10)\end{array}$ & $\begin{array}{l}12.35 \\
(6.47)\end{array}$ & $\begin{array}{c}4.05 \\
(1.19)\end{array}$ & $\begin{array}{l}14.80 \\
(6.42)\end{array}$ \\
\hline
\end{tabular}

* Arrival lag $=$ the number of fixations occurring before first arrival in the cued AOI, in the excluded AOI and in the Null AOIs. 
Table 3.

Mean (with SD) and percentage (with SD) comprehension scores by cueing condition

\begin{tabular}{lccccc}
\hline \multicolumn{1}{c}{ Comprehension } & Uncued & Entity cue & $\begin{array}{c}\text { Localized } \\
\text { coordinate cue }\end{array}$ & $\begin{array}{c}\text { Progressive path } \\
\text { cue }\end{array}$ & Total \\
\hline Kinematics & $22.82(8.59)$ & $27.07(7.29)$ & $36.89(7.27)$ & $39.60(4.45)$ & $30.73(9.93)$ \\
$\begin{array}{l}\text { precision (non } \\
\text { verbal/60) }\end{array}$ & $38.03 \%(14.32)$ & $45.11 \%(12.16)$ & $61.48 \%(12.12)$ & $66 \%(7.43)$ & $51.22 \%(16.54)$ \\
\hline $\begin{array}{l}\text { Functional mental } \\
\text { model quality }\end{array}$ & $3.89(1.93)$ & $4.28(1.87)$ & $5.56(1.90)$ & $6.60(1.90)$ & $4.94(2.13)$ \\
(verbal/15) & $25.20 \%(13.16)$ & $28.57 \%(12.45)$ & $37.10 \%(12.68)$ & $44 \%(12.67)$ & $33 \%(14.38)$ \\
\hline
\end{tabular}




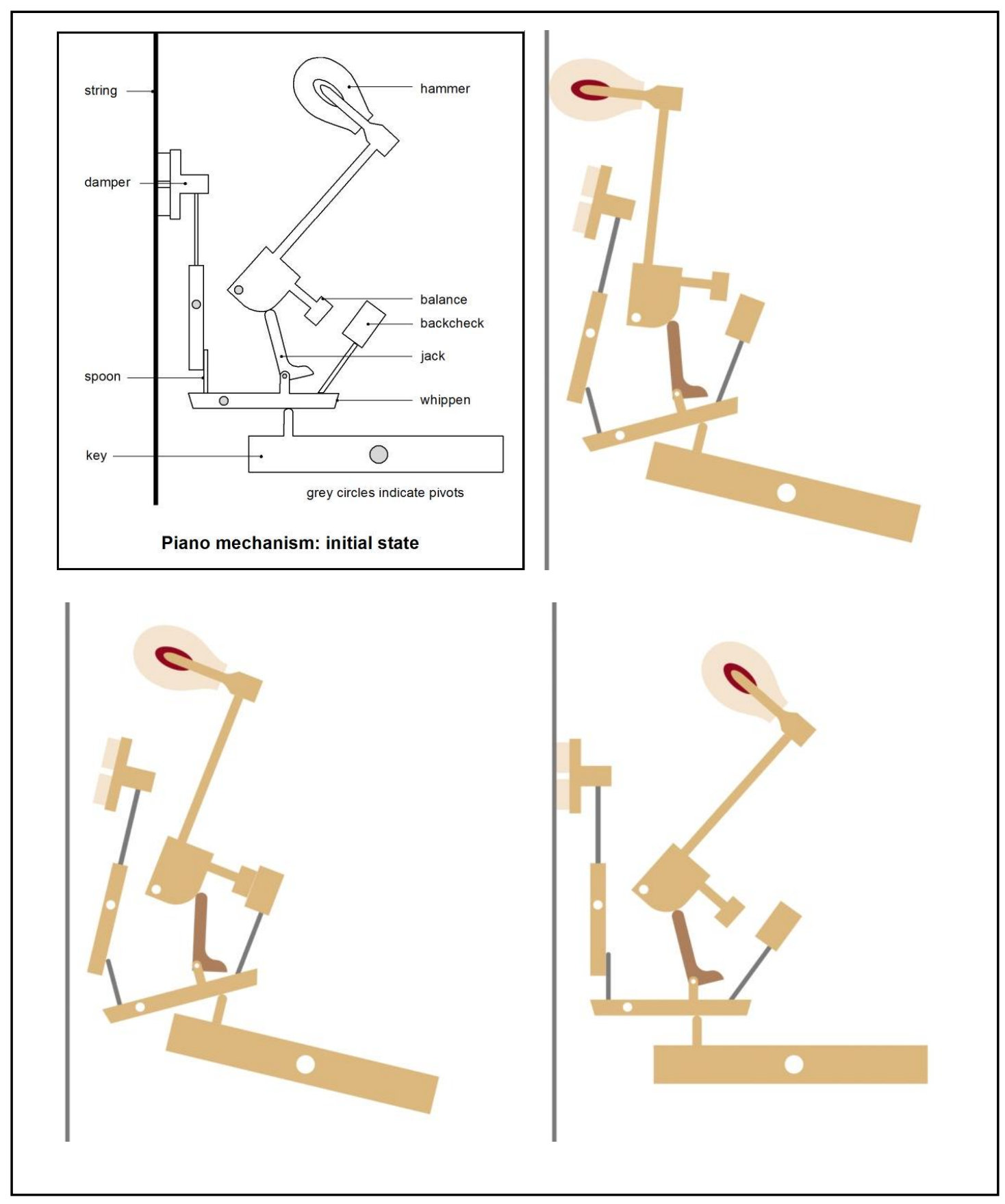

Figure 1 

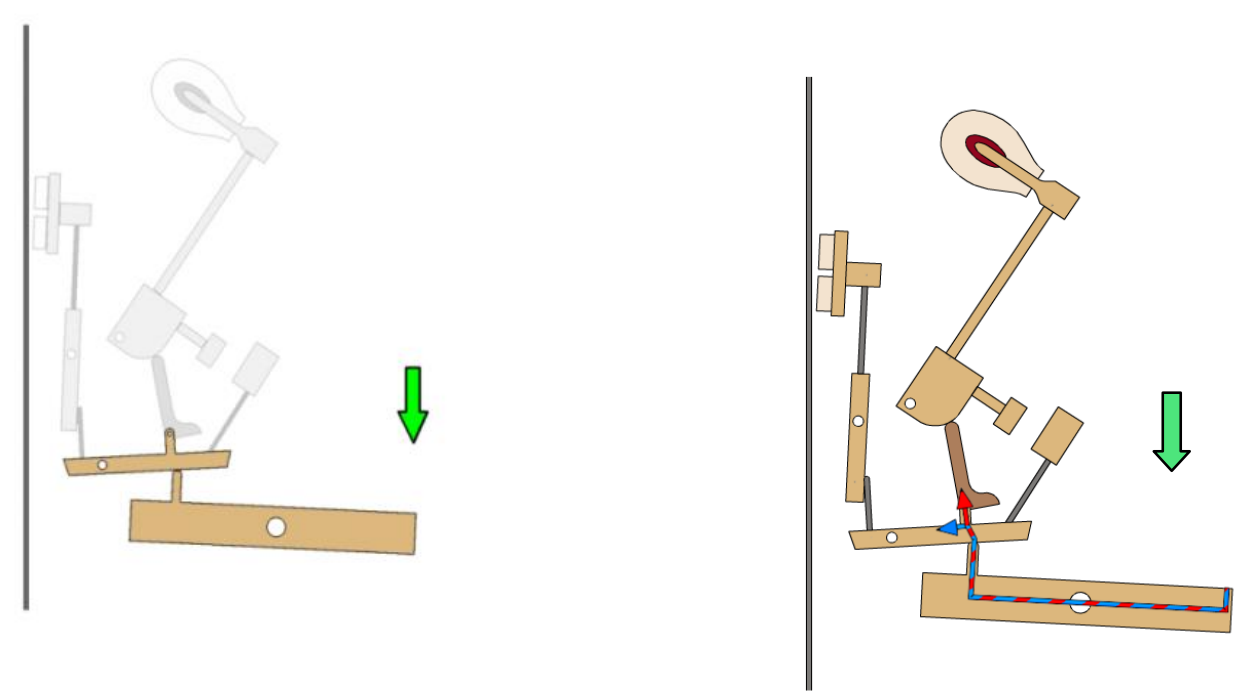

a- Entity cues

b- Progressive path cues

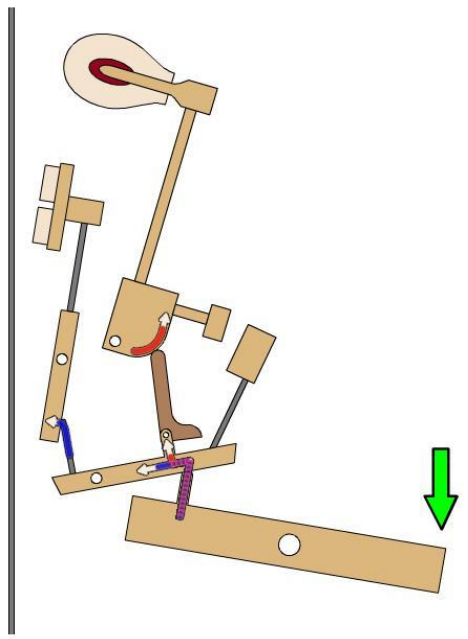

c- Local coordinated cues

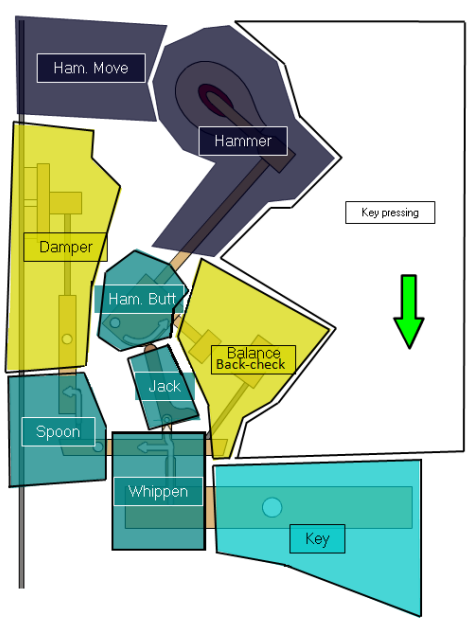

d- Initial AOIs used for eye tracking

Entity cueing was realized by decreasing progressively the colour of uncued entities. The progressive path cues pass through all the components of the piano mechanism, irrespective of their intrinsic perceptibility and thematic relevance. In local coordinated cueing, intrinsically salient entities (hammer and damper) were not cued overall but rather only with respect to their most thematically relevant parts.

Figure 2 


\section{Captions, Figures}

Figure 1. The upright piano mechanism: initial stage, striking, rebounding and resetting stages

Figure 2. Types of cueing used for the piano mechanism, and initial AOIs used for the eye tracking measures 


\section{Appendix}

\section{Appendix A}

Scoring criteria of the cross movement task

Four examples out of 30 positions of the cross

Mental Simulation task
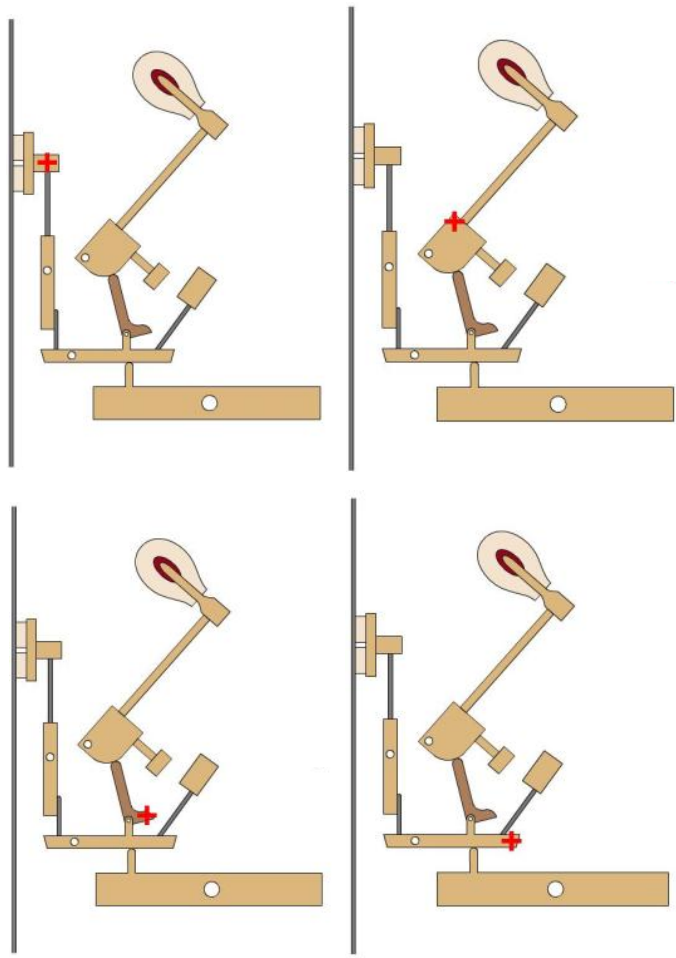

Total score $60(30 \times 2)$ : Angular direction, Amplitude

\begin{tabular}{|c|c|c|}
\hline & $\begin{array}{l}\text { Direction } \\
\text { Deviation from the } \\
\text { reference }\end{array}$ & $\begin{array}{l}\text { Amplitude } \\
\text { Deviation from the } \\
\text { reference }\end{array}$ \\
\hline 2 points & $<45^{\circ}$ & $<5 \mathrm{~mm}$ \\
\hline 1 point & $<45^{\circ}$ & $<5-7.5 \mathrm{~mm}>$ \\
\hline .5 point & $\left\langle 45^{\circ}-90^{\circ}\right\rangle$ & $</=7.5 \mathrm{~mm}$ \\
\hline 0 point & $\begin{array}{l}>90^{\circ} \\
\text { (wrong direction) }\end{array}$ & $<0-\sim>$ \\
\hline
\end{tabular}

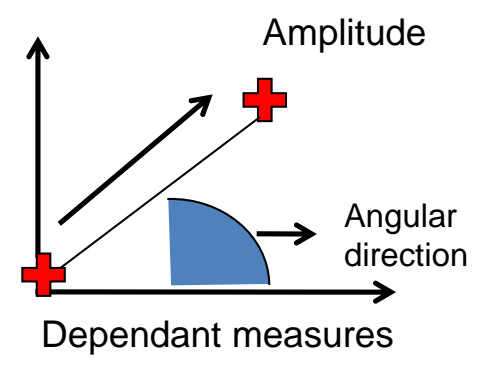




\section{Appendix B}

Scoring guide for the verbal test of mental model quality

\section{Stage 1: Striking}

1- When the key is pressed (by the pianist), it moves the key-sticker at the end of the key upwards.

2- The key-sticker raises the whippen that makes a rocking motion as a result.

3- The raising of the whippen operates the jack.

4- The upward moving jack pushes up the hammer butt.

5- The hammer-butt pivots on its axle.

6- The pivoting of the hammer-butt moves the hammer toward the string.

7- The hammer strikes the string to produce the note.

8- At the same time, the rocking motion of the whippen pushes the damper to lift it off the string.

9- The release of the damper liberates the string to sound freely when struck by the hammer.

\section{Stage 2: Recovering}

10- The hammer instantly rebounds backwards once it has struck the string.

11- The balance hammer is caught and blocked by the back-check in order to limit the hammer's backward travel. The system stays in this position as long as the key remains depressed.

\section{Stage 3: Resetting}

12- When the key is released, the whippen drops.

13- The back check releases the balance hammer.

14- The jack moves downward under the butt and the hammer returns to its initial position.

15- At the same time, the damper returns to the string. 
Appendix C. Segmentation of Strike stage for different cue types: the same three geometric configurations, Ready, Initiate, and Collide, define the start and end boundaries for segments 1 and 2 in all three cases. The implementation of cueing within these segments differs across the cueing types versions. However, in the three cueing conditions the beginning of the segment 1 was located in the key. In order to produce strictly equivalent data sets cuts off, segments were aligned on the three phases of the piano mechanism. For example, in the first stage depicted, the spoon, the hammer butt and the whippen would be loyalty-indicating AOIs in the local coordinated cues, but in the entity-cueing condition it would be key and whippen.

\section{Strike stage}

a. 'Ready' configuration (Segment 1 start)

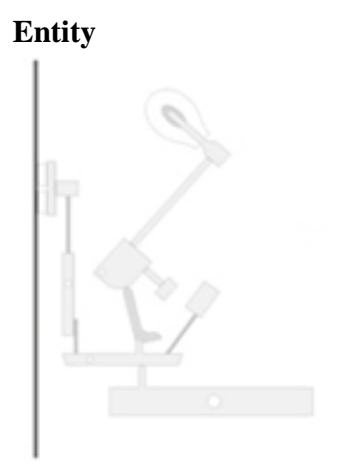

Entity cue just about to appear in key-whippen. It will persist until 'Initiate' configuration is reached

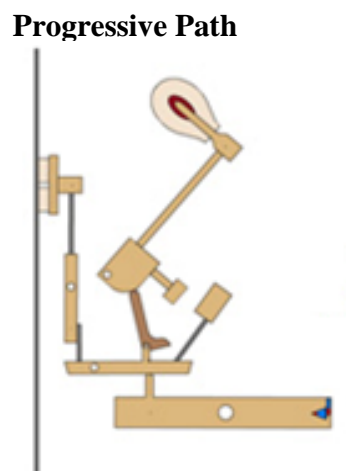

Arrowhead of cue entering extreme right side of key. Cue then travels through keywhippen

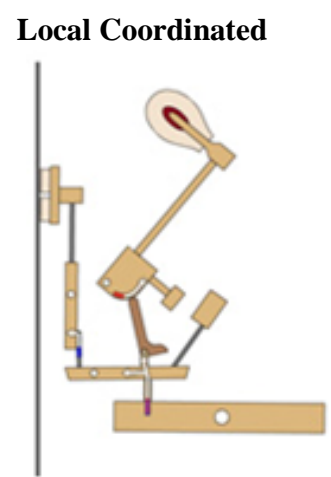

Color ribbon entering the three cued regions (key/ spoon/hammer butt b. 'Initiate' configuration (Seg. 1 end, Seg. 2 start)

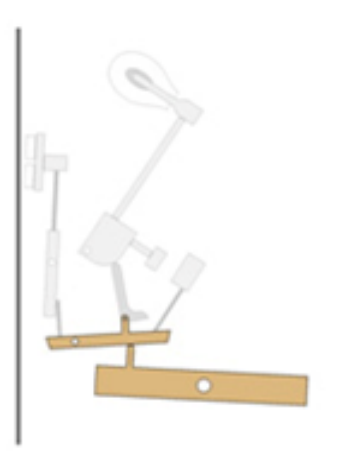

Entity cue for key-whippen just about to be replaced by entity cue for jack-damper-hammer

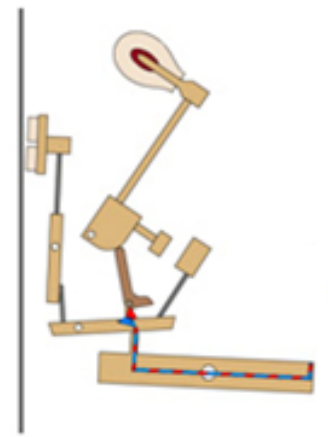

Cue has now completed its travel through key-whippen. Cues will next travel through jack-damperhammer

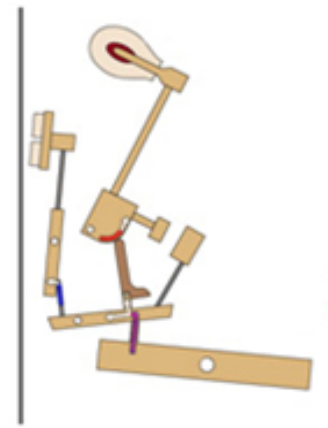

Color ribbon part-way through its traverse of the three cued regions

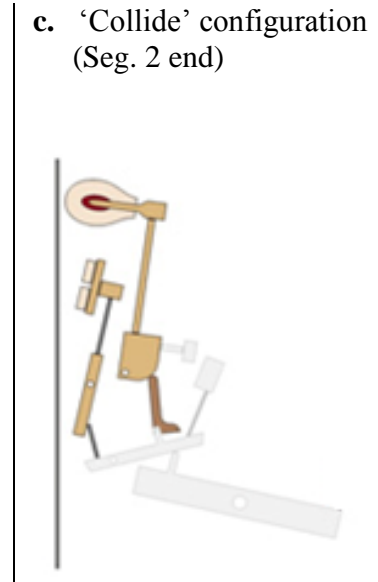

Completion of the Strike stage, just before Rebound stage begins

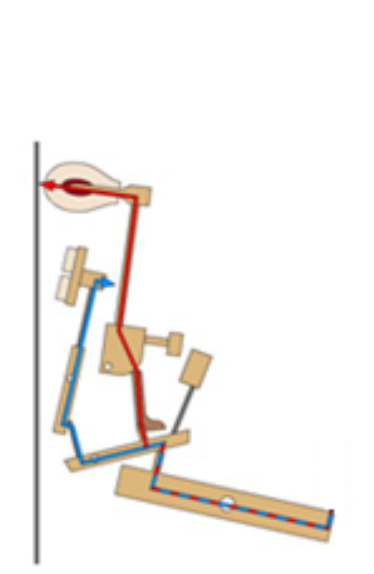

Cues have now completed their travel through jack-damperhammer

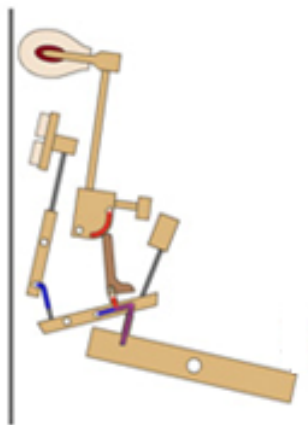

Color ribbon has completed its traverse of the three cued regions 